\title{
BEVACIZUMAB O RANIBIZUMAB, \\ O LA LEYENDA DE ROBIN HOOD Y EL REY RICARDO
}

\section{BEVACIZUMAB OR RANIBIZUMAB, OR THE TALE OF ROBIN HOOD AND KING RICHARD LION HEART}

\author{
MONÉS J ${ }^{1}$
}

Por fortuna para nuestros pacientes, sus familiares, y para los que nos hemos dedicado al tratamiento de la neovascularización coroidea, han aparecido en los últimos tiempos tratamientos mucho más eficaces. Sin embargo no era previsible, ni por el más imaginativo, la forma como se han sucedido los acontecimientos.

No hace demasiado tiempo vivíamos instalados en el beneficio del «perder menos» mediante la terapia fotodinámica para el tratamiento de la degeneración macular asociada a la edad en su forma húmeda y otras formas de neovascularización coroidea. Y ello había sido un gran avance comparado con la impotencia previa. En esa época había mucha expectativa por conocer si el advenimiento del primer fármaco antiangiogénico iba a cambiar substancialmente el pronóstico visual de nuestros pacientes. Desgraciadamente, si bien el nuevo tratamiento, el pegaptanib sódico, suponía un tratamiento más causal y con menos riesgo de casos de rebote de la agresividad de la neovascularización, los resultados visuales resultaron ser muy similares a los que se obtenían con la terapia fotodinámica. Mientras tanto, se intentaban medidas heroicas con asociaciones diversas para intentar arañar algún beneficio a los resultados del momento como, por ejemplo, la asociación con la triancinolona intravítrea.

Al mismo tiempo, estaban por conocerse los resultados de otro fármaco que estaba en investigación clínica, el ranibizumab. Nadie pensaba tampoco en ese momento que las cosas iban a cambiar demasiado. Parecía que otro fármaco antiangiogénico iba a ser más de lo mismo. Pero la sorpresa saltó en la presentación de resultados del estudio Marina en la reunión de la American Society of Retina Specialists (ASRS), en julio de 2005 en Montreal.
Por primera vez, las curvas de agudeza visual media de los pacientes rompían la línea horizontal y se situaban y mantenían en la mejoría. Inaudito e inesperado. Representaba la tercera revolución en la lucha contra la DMAE, si la terapia fotodinámica había sido la primera y la aparición del pegaptanib sódico la segunda.

A partir de este momento, y por primera vez en la historia del tratamiento de las formas exudativas o neovasculares de la degeneración macular asociada a la edad, el promedio de las visiones de los pacientes tratados fue a la ganancia. El beneficio a partir de entonces ya no sería perder menos, sino no perder, y en un buen número de casos hasta ganar visión. Si antes se aceptaban porcentajes de evitar pérdida moderada de entre 60 y $70 \%$, en ese momento pasaba a ser del $95 \%$, y lo que se lograba ahora en un $70 \%$ era no perder nada de visión. Se lograron mantener visiones al final del estudio por encima de $20 / 40$ en un $40 \%$ de pacientes, y se lograron mejorías de 3 líneas o más en porcentajes similares, resultados impensables poco tiempo atrás. Por lo tanto, estos resultados cambiaron el paradigma de eficacia a otra dimensión, a la de no perder, y a la de ir a buscar la ganancia de visión. Además, ya no era necesario distinguir subtipos para los cuales el tratamiento era más eficaz: el ranibizumab se mostraba igualmente útil en las diferentes formas de presentación de la enfermedad.

Pero al mismo tiempo sucedió otro hecho también inesperado y posiblemente jamás visto. Y aquí apareció el Robin Hood de nuestra historia. Un fármaco diseñado para otro uso diferente al intraocular, el bevacizumab - hecho además por la misma compañía farmacéutica—, iba a empezar a ser utilizado en substitución adelantada del ranibizumab,

\footnotetext{
1 Oftalmólogo Retinólogo. Institut de la Màcula i de la Retina. Centro Médico Teknon. Barcelona. España.

E-mail: jmones@institutmacularetina.com
} 
aprovechando que este último no estaba disponible. Mientras el «buen» Rey Ricardo Corazón de León luchaba en las cruzadas lejos de su tierra, el «humilde fuera de la ley» Robin Hood hacía la función del Rey en ausencia del mismo, luchando contra las «injusticias» del poder establecido del momento. Estaríamos hablando de una cuarta revolución.

Nuestro colega Phil Rosenfeld, del Bascom Palmer Institute de Miami, observó que con el uso sistémico por vía endovenosa en pacientes afectados de cáncer, y que a la vez tenían degeneración macular asociada a la edad, mejoraba la enfermedad ocular. Ello animó a este investigador y sus colaboradores a utilizar este medicamento por vía endovenosa para el tratamiento de la DMAE a pesar de los riesgos no despreciables a nivel de efectos secundarios sistémicos. Se observó la eficacia del tratamiento pero había que pagar un precio alto en cuanto al riesgo de sufrir complicaciones graves cardiovasculares, en pacientes ya de por si de edad avanzada. Estas razones les impulsaron a utilizar este medicamento directamente dentro del ojo, de forma similar al pegaptanib o al ranibizumab, a pesar de que no había sido, en principio, diseñado para tal uso. Con mucha cautela se utilizó en un inicio, pero rápidamente su uso se extendió por todo el mundo, al observarse beneficios en apariencia en una dimensión similar al ranibizumab, y que superaban a los resultados de los fármacos disponibles a pesar de ser un fármaco en uso «off-label» o diferente por el cual había sido aprobado. La «evidencia» a nivel de cientos de usuarios y el beneficio observado en sus pacientes a corto plazo condicionó, por la necesidad, una precoz y cierta «normalización» de su uso. Y de esta forma Robin Hood enamoró a la dulce Marian que en nuestra historia es la mácula.

Sin embargo, tras la aprobación del ranibizumab las condiciones cambian y la situación deja de ser simple. A partir de este momento, existe un fármaco con una eficacia muy alta basado en la evidencia de varios ensayos clínicos en fase III (Marina, Anchor, Pier). Con un fármaco altamente útil, y registrado para uso intraocular, empieza a ser más complicada la utilización del bevacizumab, tanto por cuestiones científicas como legales. Cuenta la versión de la leyenda, al menos la más extendida, que al regreso del Rey Ricardo Corazón de León, Robin Hood le rindió pleitesía. El Rey, a su vez, le entrego en matrimonio a la dulce Marian y le retiró de su vida fuera de la ley. De la misma forma, el uso del bevacizumab, justificado en ausencia del ranibizumab, deja en este momento de tener el sentido que tenía antes.

Es innegable el beneficio que el bevacizumab ha tenido para miles de pacientes. Cuesta de creer que de un día para otro el bevacizumab pueda pasar a ser un fármaco proscrito, teniendo en cuenta la evidencia, que aunque de menos nivel, es muy extensa. Queremos pensar que, sin cuestionar el reinado del Rey Ricardo, Robin estará a su lado, llegando donde no llegue el Rey. En este sentido, nos imaginamos el uso del bevacizumab, subordinado a un uso mayor del ranibizumab, pero sin su desaparición. La experiencia de estos dos últimos años no lo hacen posible. Sería deseable una cierta cohabitación, al menos hasta que tengamos los resultados del Ensayo Clínico comparativo que este verano empieza coordinado por el Dr. Daniel Martin y organizado por el National Eye Institute de Estados Unidos.

El camino ha mostrado grandes progresos, pero aun queda mucho por avanzar. El siguiente paso será conseguir fármacos con resultados de eficacia similares pero con sistemas de administración más seguros (por ejemplo inyecciones intravítreas cada 6 meses en lugar de cada mes como en la actualidad), o conseguir fármacos de liberación transescleral evitándose así las inyecciones intravítreas y sus riesgos asociados. Algunos de estos fármacos en este momentos están en estudio, y probaremos en breve en ensayos clínicos en fase III, como el VEGF trap y el bevasiranib. Supuestamente, son inhibidores más potentes del VEGF, pero sólo los resultados de los ensayos clínicos validarán esta supuesta ventaja. Además, esto no ha hecho más que empezar. La vía de la inhibición del VEGF es solamente la primera línea de investigación. En la actualidad, se están explorando nuevas vías involucradas en el desencadenamiento de la neovascularización.

En cualquier caso, y de momento, aprovechemos el avance notorio que esta área de la medicina ha experimentado recientemente y que nos ha aportado fármacos de mucha mayor eficacia y beneficio para nuestros pacientes, en el tratamiento de las formas exudativas de la degeneración macular asociada a la edad y, por extensión, en el de otras formas asociadas a otras enfermedades como miopía patológica, estrías angioides, coroiditis multifocal, etc. 